\title{
SmartCAC: Call Admission Control scheme to guarantee QoS for Voice over IEEE 802.11 WLANs
}

\author{
Prasanna Shete \\ K. J. Somaiya College of Engineering \\ Mumbai, India
}

\author{
R. N. Awale \\ V. J.T. I \\ Mumbai, India
}

\begin{abstract}
Most of the wireless LANs deployed today are IEEE $802.11 \mathrm{~b} / \mathrm{g}$ compliant. Since IEEE 802.11 standard specifies a contention based MAC- DCF, it cannot support QoS requirements of real-time traffic like voice or video. Thus these WLANs are not suitable for supporting real-time voice traffic. In this paper we propose an admission control scheme "SmartCAC", which without modifying the basic access mechanism of IEEE 802.11 tries to guarantee QoS for voice traffic. SmartCAC is a channel BW allocation scheme; in which most of the available BW is reserved for real-time voice traffic and remaining small amount is allocated to non real-time data traffic. Moreover, the BW allocation is not static; rather it can be dynamically changed depending on traffic conditions. If the voice traffic is lightly loaded then rather than wasting the greater BW share allocated to voice, it is made available for non real-time traffic sources momentarily. Our ns-2 simulations result show that the proposed SmartCAC scheme can support strict QoS requirements such as high throughput and low delay $(<150 \mathrm{~ms})$ required for voice over IP.
\end{abstract}

\section{General Terms}

Wireless Networks

\section{Keywords}

IEEE 802.11, wireless Local Area Network (WLANs), Quality of service (QoS), Medium Access Control (MAC), Bandwidth (BW).

\section{INTRODUCTION}

These days there is a widespread acceptance to WLANs for Internet access. Due to its easy and low cost deployment, IEEE 802.11 [1] has become the de-facto standard for WLANs. Moreover, there is a growing demand for VoIP for making long distance voice calls, as it provides economical call tariff as compared to PSTN or GSM. To support VoIP traffic, network should comply with ITU-T G.114 [2] recommendations which suggest that, for supporting real-time traffic the packet loss should be within 1 to $3 \%$ and transmission delay should be less than $150 \mathrm{~ms}$. VoIP works fine over wired networks but if it is to be used over wireless LANs, provision should be made to support QoS requirements of real-time voice traffic. IEEE 802.11 MAC defines two access methods; the mandatory Distributed Coordination Function (DCF) and optional Point Coordination Function (PCF). PCF is meant to support time bound services, but is available only in infrastructure mode and not supported in all 802.11 based WLANs. So we restrict our discussion only for DCF. DCF is suitable for non real-time traffic but because of introduction of large delays it cannot be used to support real- time voice traffic. Also in WLANs, the real-time voice and non real-time data flows are multiplexed on the same channel. So, as the non real-time traffic increases, it eats out the bandwidth resources of real-time traffic thus degrading the QoS of real-time traffic. Therefore, in order to support QoS for real-time traffic on WLANs, these issues need to be resolved. Various research proposals $[3,6,7,8]$ address to solve these issues, and attempt to support QoS in IEEE 802.11 based WLANs. IEEE 802.11e standard [3] defines Enhanced Distributed Channel Access (EDCA) mechanism. Four access categories (ACs) are defined for different types of traffic. The medium access is differentiated by using different inter frame spaces (IFSs) for these ACs. But this only provides service differentiation and no QoS is guaranteed. Also as the IEEE 802.11 DCF has changed drastically, so to implement this scheme requires modification/replacement of the existing IEEE 802.11 hardware. Hence the implementation is not economical. Schemes proposed in $[6,7,8]$ suggest either assigning priority to incoming real-time and non real-time traffic or service differentiation using multiple queues and hence cannot support the QoS requirements of voice traffic.

The studies conducted by Zhai et.al $[4,5]$ show that, in DCF the strict delay and delay jitter requirements can be statistically guaranteed if the instantaneous aggregate traffic contending for the shared channel is controlled below network capacity. Under this condition WLAN operates at the optimal point where the collision probability is small and MAC delay is quite small to support voice traffic. If the incoming traffic load is greater than the threshold, WLAN enters saturation, the delay increases significantly and throughput falls drastically. In contrast, if WLAN is lightly loaded the channel bandwidth is wasted. So to tune the contention based network to operate at the optimum point, an effective and efficient mechanism is required that will regulate the incoming traffic. In this paper we propose an admission control scheme "SmartCAC", that can provide QoS guarantees for voice traffic over WLAN. SmartCAC will be a software upgrade for the IEEE 802.11 WLANs.

The remaining paper is organized as follows; section 2 describes the overview of SmartCAC, its design and the definition of the term channel busyness ratio which acts as the network status indicator is given in section 3. SmartCAC mechanism is explained in section 4 . The simulation configuration in ns-2 [9], and performance analysis of SmartCAC is presented in section 5. Section 6 concludes the paper. 


\section{SmartCAC OVERVIEW}

Our SmartCAC scheme can be explained as follows: SmartCAC scheme determines when and how the packets are to be passed from higher layer to the MAC layer to contend for shared channel. The admission decision is based on the availability of BW resources required for the flows. It acts as the as a control entity on top of the MAC sublayer. The status of network is measured with the help of Channel Busyness Ratio [4]. SmartCAC is able to provide statistical QoS guarantees for real-time traffic. Also it allows the non realtime traffic to utilize the remaining channel capacity left out by the real-time traffic, without affecting their QoS level, thereby enabling the network to approach the theoretical maximal channel utilization.

\section{DESIGN METRICS}

Here we define the design metrics used and discuss how channel busyness ratio can be used to represent the network status of IEEE 802.11 WLAN.

Channel busyness ratio $R_{b}$ : It is defined as the ratio of time the channel is busy to the total time. Busy time corresponds to time for successful transmissions as well as collisions.

Channel utilization ratio $R_{s}$ : It is the ratio of successful transmission periods to the total time. It counts every period $T_{\text {suc }}$ with a successful transmission, which includes time for RTS, CTS, DATA, ACK and all necessary inter frame spaces i.e. SIFS and DIFS. $R_{b}$ can be easily calculated using the physical and virtual carrier sensing mechanism of IEEE 802.11 CSMA based MAC. The channel is busy when the measuring node is sending, receiving or its NAV indicates channel is busy, otherwise channel is considered idle.

From the results, of work conducted by Zhai et al. [4], it can be seen that there is an optimal point for IEEE 802.11 DCF, which corresponds to certain amount of arriving traffic. At this optimal point MAC protocol can satisfy the strict QoS requirements of real-time traffic and achieve maximal channel utilization. Figure 1 presents ns- 2 simulation results of [4] that illustrate the performance of throughput, delay and delay variation as a function of channel busyness ratio when RTS/CTS is used. Table 1 summarizes the IEEE 802.11 system parameters.

Table 1. IEEE 802.11 System Parameters

\begin{tabular}{|l|l|}
\hline Parameter & Value \\
\hline Bit rate for DATA packets & $2 \mathrm{Mbps}$ \\
\hline Bit rate for RTS/CTS/ACK & $1 \mathrm{Mbps}$ \\
\hline PLCP Data rate & $1 \mathrm{Mbps}$ \\
\hline Backoff Slot Time & $20 \mu \mathrm{s}$ \\
\hline SIFS & $10 \mu \mathrm{s}$ \\
\hline DIFS & $50 \mu \mathrm{s}$ \\
\hline PHY header & 192 bits \\
\hline MAC header & 224 bits \\
\hline DATA packet & 8000 bits + PHY header \\
+ MAC header
\end{tabular}

Every node initiates an identical UDP/CBR traffic flow to a randomly selected neighbor node. Different points in the Fig. 1 correspond to different sending rates of flows. As can be seen from the graphs, there is a turning point in all the curves where the channel busyness ratio is about 0.95 . Before this point, as the input traffic increases the channel busyness ratio increases and the throughput also increases linearly with $R_{b}$, the delay (including queuing delay, channel contention time or backoff time and transmission time) and delay variation only slightly increase and are small enough to support the real-time traffic. After this point, the throughput drops quickly, and the delay and delay variation increase rapidly. Thus, this turning point is the optimal point that we should select the network to operate, where the throughput is maximized and, delay and delay variation are small. When the WLAN operates at the optimal point, there is almost no possibility of collisions and $\mathrm{R}_{\mathrm{b}} \equiv \mathrm{R}_{\mathrm{s} \text {. }} \mathrm{R}_{\mathrm{b}}$ is stable around 0.9 (without RTS/CTS) or 0.95 (with RTS/CTS) independent of packet size or number of users [4].

Let $\mathrm{B}_{\mathrm{U}}$ denote the channel utilization corresponding to the optimal point. Since, $\mathrm{R}_{\mathrm{b}} \equiv \mathrm{R}_{\mathrm{s} ;} \mathrm{B}_{\mathrm{U}} \equiv 0.95$ or 0.90 (depending upon whether RTS/CTS is used or not) independent of packet size or number of active nodes. By keeping track of the channel busyness ratio, we can know the network status. Also channel busyness ratio can be used to regulate the input traffic to support QoS. The admission control scheme should maintain $\mathrm{R}_{\mathrm{b}}$ close to $\mathrm{B}_{\mathrm{U}}$ to guarantee both, a good QoS level and high aggregate throughput.

\section{SmartCAC MECHANISM}

SmartCAC is a dynamic admission control scheme that admits or rejects new traffic flow on availability of BW resources and tries to guarantee a QoS level for the admitted traffic. Admission decision is taken by the point coordinator of WLAN for each traffic flow. A new traffic flow is admitted only if the requested amount of $\mathrm{BW}$ resource is available. Initially we reserve $75 \%$ of total available BW utilization $\left(\mathrm{B}_{\mathrm{U}}\right)$ for real-time voice traffic and remaining $25 \%$ for non realtime data traffic. This reservation is not static rather it can be dynamically adjusted depending upon traffic composition. If no real-time traffic is arriving from higher layer, rather than wasting the reserved $\mathrm{BW}$, it can be allocated to non real-time traffic momentarily. Let $B_{R T}$ denote the share of the bandwidth for real-time voice traffic hence, $\mathrm{B}_{\mathrm{RT}}=0.75 * \mathrm{~B}_{\mathrm{U}}$. And let $\mathrm{B}_{\mathrm{NRT}}$ denote the share of bandwidth for non real-time traffic hence, $\mathrm{B}_{\mathrm{NRT}}=0.25 * \mathrm{~B}_{\mathrm{U}}$. This ensures maximum channel resources for real-time voice traffic, at the same time non real-time traffic remains operational all the time since it is allotted with some part of channel resources.

We model the voice traffic as VBR (variable bit rate) and background data traffic as CBR (constant bit rate). Three parameters viz; $R, R_{\text {peak }}$ and len are used to characterize the bandwidth requirements of the traffic flows, where $R$ is the average rate, $R_{\text {peak }}$ is the peak rate (both in bits/sec) and len is the average packet length in bits. For CBR traffic, $R=R_{\text {peak }}$ and for VBR, $R<R_{\text {pea. }}$. For admission control, these parameters of voice flows are converted into channel utilization parameter ' $V_{U}$ ' (i.e. the channel time a voice flow will occupy) as:

$$
V_{U}=\frac{R}{\text { len }} * T_{\text {suc }}
$$

And

$$
V_{\text {peakU }}=\frac{R_{\text {peak }}}{\text { len }} * T_{\text {suc }}
$$

Similarly for data flow, if ' $D_{u}$ ' denotes the channel utilization, we can have:

$$
D_{u}=\frac{R}{l e n} * T_{\text {suc }}
$$




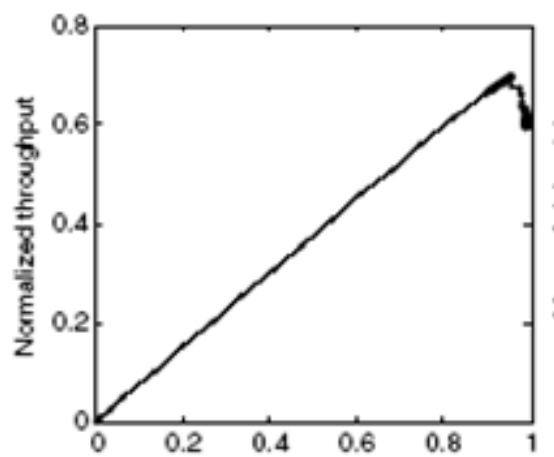

(a) Channel busyness ratio



(b) Channel busyness ratio

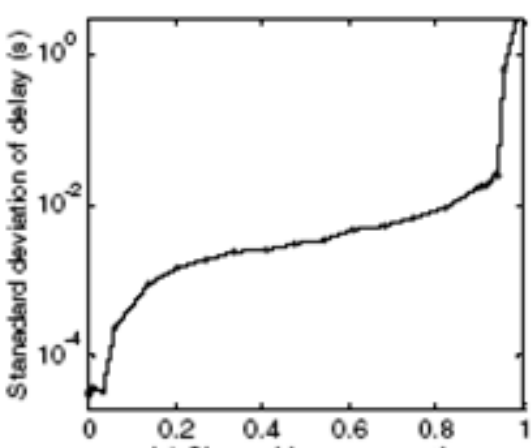

(c) Channal busyness retio

Fig. 1. Throughput and delay performance with 50 nodes, channel busyness ratio vs: a) normalized throughput, b) mean of delay (s), c)standard deviation of delay (s) [4]

Where, $\underline{T}_{s u c}$ is the transmission time of one packet, including RTS, CTS, Data and ACK and all the necessary inter-frame spaces i.e. DIFS, SIFS defined in [1].Therefore,

$$
T_{S u c}=D a t a+A C K+R T S+C T S+3 * S I F S+D I F S
$$$$
\text { (with RTS/CTS) }
$$

$$
T_{\text {Suc }}=\text { Data }+A C K+S I F S+D I F S(\text { without RTS/CTS })
$$

Thus $\left(V_{U}, V_{\text {peakU }}\right)$ specify voice flow's bandwidth requirement and $\left(D_{u}\right)$ specifies data flow's bandwidth requirement.

At the coordinator/AP, the total bandwidth occupied by all admitted real-time flows is recorded in two parameters, called the aggregate $\left(V_{U}, V_{\text {peak } U}\right)$ denoted by $\operatorname{Agg}\left(V_{U}, V_{\text {peak }}\right)$ and the total bandwidth occupied by all admitted non real-time data flows is recorded as aggregate $\left(D_{u}\right)$ denoted by $\operatorname{Agg}\left(D_{u}\right)$. They are updated when a flow joins or leaves the network through the following procedure.

When a node wants to establish a flow, it must convert the bandwidth requirement into the form of $\left(V_{U}, V_{\text {peakU }}\right)$ or $\left(D_{u}\right)$, and send a request with this requirement, to the $\mathrm{AP} /$ coordinator. Upon receiving a request with these parameters, the $\mathrm{AP} /$ coordinator examines whether there are enough resources to accommodate the new flow i.e. whether the remainder quota of $\mathrm{B}_{\mathrm{RT}} \& /$ or $\mathrm{B}_{\mathrm{NRT}}$ can accommodate the new traffic flow by carrying out the following procedure:

For real-time voice traffic:

If $\left[A g g\left(V_{U}\right)+V_{U}\right] \leq B_{R T} \&\left[A g g\left(V_{\text {peakU }}\right)\right] \leq B_{U} \quad$ the $\quad$ AP issues connection admitted message, and updates the value of $\operatorname{Agg}\left(V_{U}, V_{\text {peakU }}\right)$ with

$\left.\left[A g g\left(V_{\text {peakU }}\right)+V_{\text {peakU }}, A g g\left(V_{U}\right)+V_{U}\right)\right]$

Otherwise AP issues connection-rejected message.

For non real-time data traffic:

If $\left[A g g\left(D_{u}\right)+D_{u}\right] \leq B_{N R T}$, AP issues a connection admitted message and updates $\operatorname{Agg}\left(D_{u}\right)$ with $\left[A g g\left(D_{u}\right)+D_{u}\right]$

Else if, $\left[A g g\left(D_{u}\right)+D_{u}\right] \leq B_{R T}, \quad$ AP admits the flow momentarily for a predefined time period. The flow is dropped after elapsing of the predefined time period.

Otherwise AP issues connection-rejected message.

When the flows end, the source nodes of the flow should send a "connection terminated" message to the AP/coordinator.
The AP/coordinator responds with a "termination" confirmed message and updates $\operatorname{Agg}\left(V_{U}, V_{\text {peakU }}\right)$ or $\operatorname{Ag}\left(D_{u}\right)$ respectively.

\section{PERFORMANCE EVALUATION}

In this section, we illustrate through simulations that, SmartCAC can provide QoS guarantee for voice traffic. The simulation results show that, with the proposed scheme higher throughput and very small delay can be achieved in IEEE 802.11 WLAN as compared with the vanilla 802.11 WLAN.

\subsection{Simulation setup}

We have simulated IEEE 802.11 based WLAN using the network simulator ns-2.30, with 50 mobile nodes moving in $500 * 500 \mathrm{~m}$ topology. In the simulations, the channel rate is $2 \mathrm{Mbps}$ and the simulation is run for $120 \mathrm{sec}$. The queue-length at each node is 50 packets. System parameters of IEEE 802.11 , used in the simulations are given in Table 1.

We use two different classes of traffic, to model real-time voice and non real-time data traffic.

Voice Traffic model: The voice traffic is modeled as variable bit rate (VBR) traffic using the on/off source model, with exponentially distributed on and off periods of $300 \mathrm{~ms}$ average each. During the on periods, traffic is generated at a rate of $32 \mathrm{kbps}$ with a packet size of 160 bytes, therefore the inter packet time is $40 \mathrm{~ms}$ and during the off periods, no traffic is generated.

Data traffic model: Data traffic is modeled as CBR with a rate of $64 \mathrm{kbps}$ and packet size of 1000 bytes, thus inter packet time is $125 \mathrm{~ms}$.

In our simulations, we have used the RTS/CTS mechanism. The traffic load is gradually increased by periodically adding a new voice or data flow in an interleaved fashion, to observe the performance of SmartCAC and the effect of newly admitted flow on the performance of previously admitted flows. Until 96 seconds, a new voice flow is added at time instances of $4 * \mathrm{i}$ seconds $(0 \leq \mathrm{i}<25)$ and a data flow is added at 2 seconds later in the same fashion. To study the steady state performance of SmartCAC, we stop new traffic flows after 100 seconds. Trace graph [10] was used as plotting tool for the simulation trace data.

\subsection{Simulation Results}

The simulation results show that, at 96 seconds there are total 25 (20 voice and 5 data) flows admitted, rest of the 5 voice flows are rejected. Remaining data flows are given momentary access whenever the bandwidth resources allotted 
to voice flows are free, ensuring better channel utilization. As calculated by equations, (1), (2) and (3) each voice flow contributes 0.035 and each data flow contributes 0.042 to the channel busyness ratio. Hence, the available channel resources support only the above mentioned number of traffic flows and any further requests for traffic admission are rejected by the SmartCAC mechanism.

Fig. 2 (a, b) show the throughput Vs time plots of voice (VBR) and data (CBR) traffic, Fig. 2 (c) shows packet sizeVs delay of voice traffic when SmartCAC is used and Fig. 3 ( $a, b$, c) show these parameters in legacy IEEE 802.11 based WLAN without SmartCAC. As can be seen the throughput of voice traffic is slightly increased with our scheme, where as throughput of data traffic is reduced but almost constant in SmartCAC. Also, the packet size Vs average delay of voice flows is reduced by greater extent and much below the requirements for real-time flow according to the ITU-T G.114 recommendations. Table 2 shows the results and comments about the percentage improvement achieved with SmartCAC.

Table 2. Throughput and delay parameters of IEEE 802.11 WLAN with and without SmartCAC scheme.

\begin{tabular}{|l|l|l|l|}
\hline & $\begin{array}{l}\text { IEEE } \\
802.11\end{array}$ & 802.11 with CAC & $\%$ change \\
\hline $\begin{array}{l}\text { Voice (VBR) } \\
\text { throughput }\end{array}$ & $\begin{array}{l}3.0 * 10^{5} \\
\mathrm{bps}\end{array}$ & $2.5 * 10^{5} \mathrm{bps}$ & $+16.67 \%$ \\
\hline $\begin{array}{l}\text { Voice (VBR) } \\
\text { average } \\
\text { delay }\end{array}$ & $3.5 \mathrm{sec}$. & $30 \mathrm{~ms}$ & $-99.14 \%$ \\
\hline $\begin{array}{l}\text { Data (CBR) } \\
\text { throughput }\end{array}$ & $\begin{array}{l}9.0 * 10^{5} \\
\mathrm{bps}\end{array}$ & $4.0 * 10^{5} \mathrm{bps}$ & $-55.55 \%$ \\
\hline
\end{tabular}

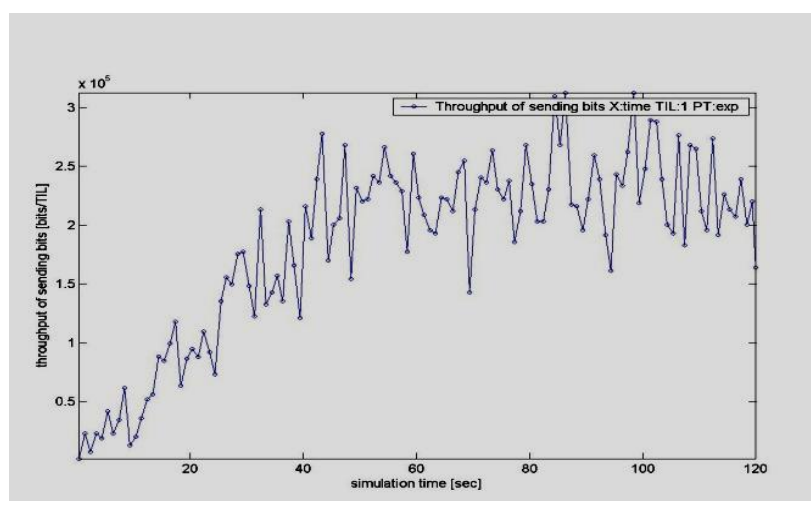

Figure 1 (a) Throughput Vs time of voice (VBR/exp) traffic with SmartCAC.

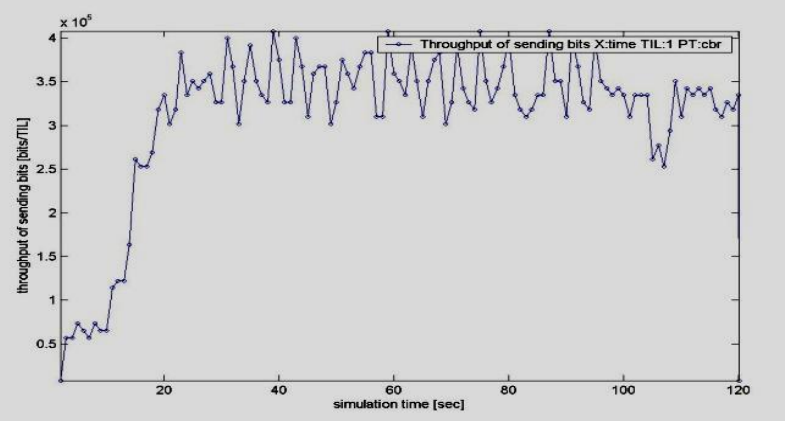

Fig. 2 (b) Throughput Vs time of data (CBR) traffic with SmartCAC.

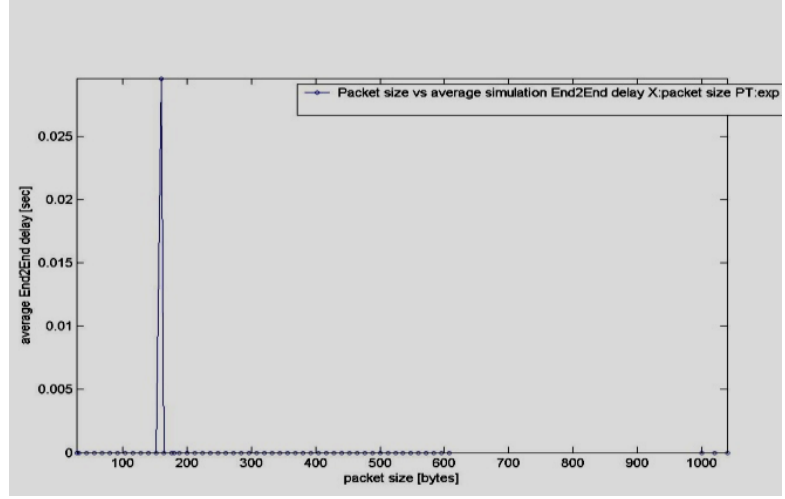

Fig. 2(c) Packet size Vs Average Delay plot of voice (VBR/exp) traffic with SmartCAC.

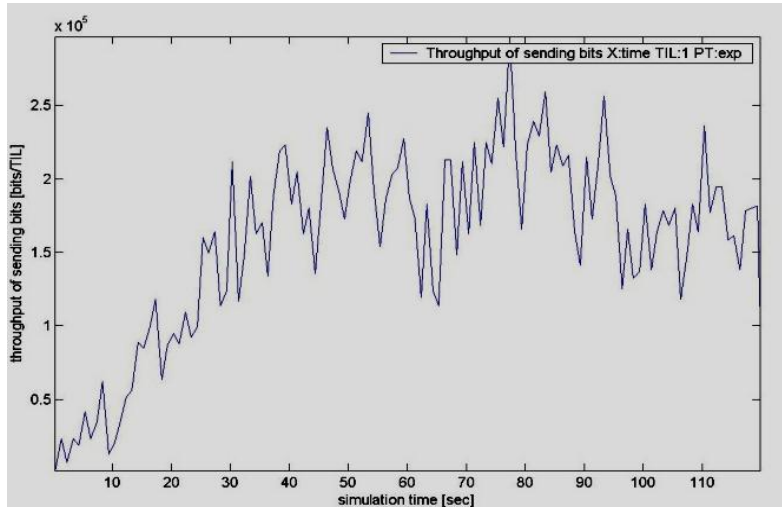

Fig. 3 (a) Throughput Vs time of voice (VBR/exp) traffic without SmartCAC

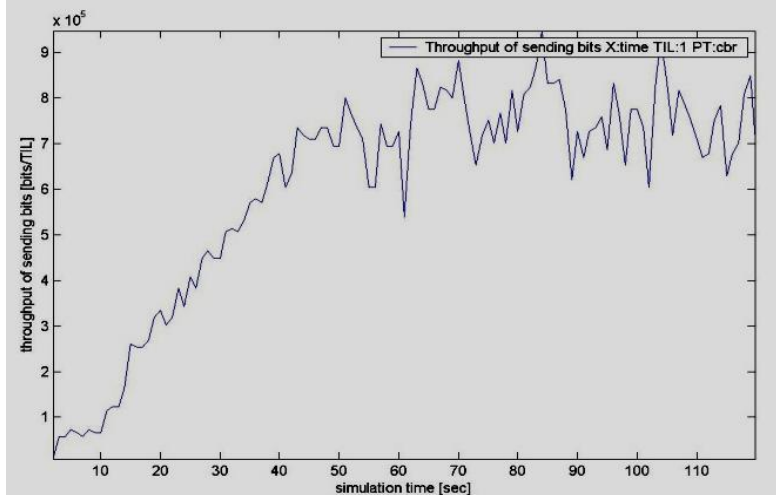

Fig. 3 (b) Throughput Vs time of data (CBR) traffic without SmartCAC

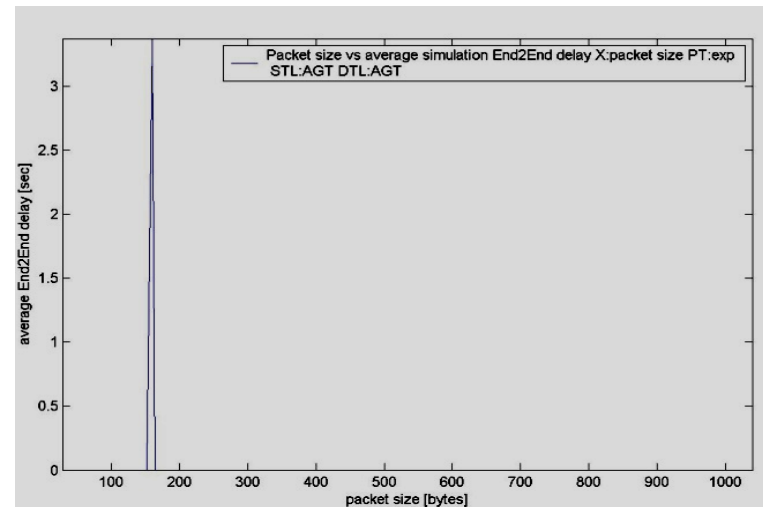

Fig. 3(c) Packet size Vs Average Delay plot of voice (VBR/exp) traffic without SmartCAC. 


\section{CONCLUSION}

In this paper we proposed a simple and effective call admission control scheme SmartCAC to support QoS for realtime traffic in the IEEE 802.11 wireless LANs. Using the channel busy-ness ratio efficietly, the proposed scheme enables the 802.11 wireless network be operated at the optimal point thereby, guaranteeing the strict QoS requirements of the real-time voice flows at the same time achieve the maximum possible channel utilization.

Here simple 2-ray ground propagation model was considered ignoring the fading mechanisms like Raleigh fading etc, which will be addressed in later work

\section{REFERENCES}

[1] IEEE standard for Wireless LAN Medium Access Control (MAC) and Physical Layer (PHY) specifications, ISO/IEC 8802-11: 1999(E) (1999).

[2] IEEE standard for Wireless LAN Medium Access Control (MAC) and Physical Layer (PHY) specifications: Medium Access Control (MAC) Enhancements for Quality of Service (QoS), IEEE 802.11e/ D13.0, July (2005).

[3] ITU-T G.114, “One-Way Transmission Time,” 1996.

[4] H. Zhai, X. Chen, Y. Fang, How Well Can the IEEE 802.11 Wireless LAN Support Quality of Service?, IEEE
Trans. Wireless Commun., vol. 4, no. 6, Nov. 2005, pp. 3084-3094.

[5] H. Zhai, Y. Kwon, and Y.Fang, Performance Analysis of IEEE 802.11 MAC Protocols in Wireless LANs, Wireless Commun. And Mobile Comp., vol. 4, Dec. 2004, pp. 917-31

[6] D. Gao, J. Cai, and K. N. Ngan, Admission Control in IEEE 802.11e Wireless LANs, IEEE Network., Special Issue on Wireless Local Area Networking: QoS Provision and Resource Management, vol. 19, no.4, July/Aug. 2005,pp. 6-13.

[7] W. Pattara-Atikom, P. Krishnamurthy, and S. Banerjee, Distributed Mechanisms for Quality of Service in Wireless LANs, IEEE Wireless Commun., vol. 10, no. 4, June 2003, pp.26-34.

[8] J. Yu, S. Choi, and J. Lee, Enhancements of VoIP over IEEE 802.11 WLAN via Dual Queue Strategy, Proc. IEEE ICC '04, Paris, France, June 2004.

[9] The network simulator ns-2. http://www.isi.edu/nsnam/ns

[10] Trace graph. $\quad$ http://www.tracegra 